\title{
Human-nature connectedness as a pathway to sustainability: a global meta-analysis
}

Gladys Barragan-Jason ( $\square$ Gladys.BarraganJason@gmail.com )

CNRS https://orcid.org/0000-0002-8002-6643

\section{Claire de Mazancourt}

CNRS

Camille Parmesan

CNRS

\section{Michael Singer}

CNRS

Michel Loreau

Station d'Ecologie Expérimentale du CNRS https://orcid.org/0000-0002-0122-495X

\section{Article}

Keywords: sustainability, human-nature connectedness, meta-analysis

Posted Date: July 2nd, 2021

DOl: https://doi.org/10.21203/rs.3.rs-567480/v1

License: (c) (1) This work is licensed under a Creative Commons Attribution 4.0 International License. Read Full License 


\section{Abstract}

Internationally-agreed sustainability goals are being missed. Here, we document a potential reason for this failure and show how the extent to which humans see themselves as part of nature - known as human-nature connectedness (HNC) - can be used as a leverage point for increasing public engagement towards sustainability targets. We conduct the first global quantitative analysis of the HNC literature, with meta-analyses of 59 experimental and 147 correlational studies. Meta-analysis of experiments documents significant increases in $\mathrm{HNC}$ after manipulations involving contact with nature and "mindfulness" practices (focusing on oneself and surroundings in the present moment). By contrast, estimated effects on HNC of environmental education and virtual nature experience were minimal. Metaanalysis of correlational studies documents that individuals with high HNC had higher levels of physical contact with nature, more engagement in mindfulness and more pro-nature behaviours. They also felt significantly happier and healthier than those with low HNC. Thus, HNC is positively linked to mindsets that value sustainability and behaviours that enhance it. Further, we show that HNC can be enhanced by interventions and we identify techniques most likely to succeed. These results suggest that enhancing $\mathrm{HNC}$, via promotion of targeted practices, can improve sustainability.

\section{Main Text}

Anthropogenic climate change and biodiversity loss are major threats not only to non- human living beings but also to our own survival ${ }^{1,3,8,9}$. Despite there being apparent international consensuses to better preserve nature (e.g., the Convention on Biological Diversity ${ }^{4}$ and Aichi Biodiversity Targets), to limit global warming to $2^{\circ} \mathrm{C}$ (e.g., the Paris Agreements) and to create a sustainable, equitable world (e.g., the Sustainable Development Goals - SDGs), actions remain limited and sustainable targets have failed to be achieved ${ }^{5,10}$. Why do citizens and governments still find it hard to consider the health and well-being of humans as dependent upon the health of the natural world ${ }^{1,3,8}$ ?

One possible explanation is cultural. Monotheist religions ${ }^{11,12}$ and influential modern philosophers ${ }^{13,14}$ advocated a discontinuity between the inner-worlds of humans and those of other living beings, which has led people to consider themselves disconnected from, and dominant over, the rest of nature (the modern "Western" worldview). Another explanation is the increased urbanization of the world's human population. Today, more than $55 \%$ of people live in urban areas, and this proportion is expected to increase to $68 \%$ by $2050^{15}$. A major consequence is the increased disconnection of people from experience of natural habitats ${ }^{16}$. The combination of both psychological and physical disconnects from the natural world can result in devaluation of nature, thereby legitimizing and facilitating destructive practices towards nature by individuals and societies (Fig. 1).

What can be done to modify these destructive trends? International scientific assessments, such as the MA, IPCC and IPBES, indicate that healthy natural systems are crucial to reach sustainability ${ }^{1-3}$. The IPBES further claims that sustainable goals will not be achieved without a "transformative change" 
including an increase in "awareness of connectivity in the environmental crisis and new norms regarding interactions between humans and nature" (Fig. 1). A key societal trait that is relevant to achieving sustainability is Human-Nature Connectedness ${ }^{12-14}$ (HNC), which has been described and defined in different, often complex, ways (Supplementary information (SI) Appendix 1). We define HNC as the extent to which humans include the natural world in the self or see themselves as part of nature. A number of recent studies found that estimates of HNC were positively correlated both with pro-environmental behaviour ${ }^{17,18}$ and with human welfare ${ }^{19,20}$.

Due to paucity of data, prior synthetic analyses of the roles of HNC were forced to lump studies with disparate methodologies, whether they be experimental or correlational, and whether or not they used metrics of HNC that had been validated by methods that we describe in SI Appendices 2 and 3. Here, we take advantage of the rapidly-increasing literature to restrict our database to studies using at least one of the validated quantitative metrics of $\mathrm{HNC}^{13,14,19,20-23}$ and to separate experimental from correlational studies. Thus, we provide, for the first time, a robust, coherent and global synthesis of the HNC literature with separate meta-analyses of experimental and correlational studies (Extended data Fig. 1 and Extended data Table 1). This allows us to identify practices that have the potential for increasing HNC, and investigate whether HNC is a key element of sustainability, in particular nature conservation and human welfare.

\section{Experimental studies}

Existing experimental studies are strongly biased towards adults and towards high-income, industrialized countries with a "Western" worldview (Extended data Fig. 2). Among them, we identified 6 types of experimental designs: (1) exposure to real nature: direct contact with nature, either outdoors or indoors; (2) exposure to virtual nature: videos or pictures of nature; (3) mindfulness: focusing one's attention on one's inner self and one's environment in the present moment; (4) environmental education: exposure to naturalist, scientific and ecological knowledge of the natural world; (5) combination of exposure to real nature + environmental education and, (6) combination of exposure to real nature + mindfulness.

We combined two types of comparison: (1) sequential comparison of trait measurements made on the same participants before and after experimental treatment and (2) simultaneous comparison of control and treatment groups (see Methods and SI Appendix 4).

All experimental designs, except environmental education, affected HNC significantly and positively (Fig. 2 and Extended data Table 2). The clearest positive effects on HNC were those of mindfulness, with or without experience of real nature, while estimated positive effects of environmental education were low. These analyses of experimental studies show that exposing individuals to nature, either real or virtual, does improve HNC. They also suggest exciting lines of future research to improve the efficiency of experimental programs designed to raise $\mathrm{HNC}$ by including mindfulness practices and/or by trying less anthropocentric transmission of scientific information. 
Like experimental studies, correlational studies are strongly biased toward adults from industrialized countries. Meta-analysis of their results shows that $\mathrm{HNC}$ is negatively correlated with materialism/consumerism and political conservatism and positively correlated with the following suite of behaviours, opinions and personality traits: naturalist knowledge; time spent in natural outdoor spaces; engagement in mindfulness practices; pro-environmental values; humanistic values; happiness and good health.

In sum, individuals with high HNC had deeper knowledge of nature, spent more time in natural outdoor spaces, engaged more in mindfulness practices and were happier and healthier than those with low HNC. It is not surprising, then, that high HNC individuals displayed more pro-environmental values, expressed as concern and attitudes toward the ecological environment. They were also more humanistic, in the sense of more strongly expressing their moral responsibilities to other humans, and their sense of being part of communities within society (Fig. 2; see Methods summary, Methods and SI Appendix 4 for detailed categories and Extended data Table 3, Extended data Figs. 3 to 6).

Additionally, one-third of the correlational papers (26 out of 108) addressed causal hypotheses using either structural equation models or path analysis. Although these approaches have limited ability to infer causality, these studies nonetheless suggest impacts on HNC of both contact with nature and mindfulness, in agreement with results from experimental data (Fig. 2 and Extended data Table 2). In the opposite direction of causality, they also suggest impacts of HNC on pro-environmental values, human well-being and health and pro-environmental behaviours.

Taken together, correlational data support conclusions from experimental studies but also add to them, showing HNC to be positively linked to values and behaviours that enhance ecological sustainability and well-being, and negatively linked to non-environmental or anti-environmental values. Future experimental research is needed to test causal relationships, particularly the hypothesis suggested by our metaanalysis that raising HNC would enhance both human welfare and conservation of nature.

\section{Implications for Policy.}

Our meta-analyses robustly show that the extent to which people feel part of the natural world can be enhanced by very simple interventions involving contact with nature and mindfulness practices, at least for industrialized cultures that form the bulk of studies. They also show that validated HNC indices are positively linked to human welfare and nature conservation. Thus, improving HNC through contact with nature and mindfulness can be a valuable way to help individuals to understand and experience how much human welfare and nature conservation are interconnected ${ }^{21}$. As an example, "greening" schoolyards could improve citizens' HNC and well-being and thereby foster support for sustainable policies, adding to its known positive effects on urban biodiversity and climate adaptation (the virtuous circle in Fig. 1).

Surprisingly, we found little impact of environmental education on HNC, and we hypothesize that this is due to the traditional anthropocentric transmission of scientific knowledge. While this result remains to 
be further tested, studies in non-industrialized populations ${ }^{28}$ and children ${ }^{29}$ suggest that modifying the way scientific information is transmitted using non-anthropocentric knowledge about non-human species can have a positive impact on HNC. Examples of non-anthropocentric approaches include focusing on similarities between humans and other species ${ }^{22-26}$ and taking the perspectives of other species and empathizing with them ${ }^{27}$.

If we are to develop efficient research and conservation programs aimed at simultaneously protecting humans and nature worldwide ${ }^{21}$, we urgently need longitudinal studies in children and in non-industrial traditional cultures. Children from industrialized societies are known to show a strong affinity for nonhumans ${ }^{29}$, which tends to fade with age $\mathrm{e}^{30,31}$ and develop into low ecological concern at adulthood ${ }^{32,33}$ along with the acquisition of anthropocentric cultural norms ${ }^{34}$. We know much less about equivalent developmental changes in traditional societies, although pro-environmental behaviours may be more likely to persist into adulthood in societies with a high interdependency between humans and nature ${ }^{28}$. Accumulation of knowledge about cultural and developmental norms and values in diverse societies, both traditional and industrial, should assist in building international environmental and educational policies and empowering citizens and governments to take actions and achieve sustainable targets at a global scale.

\section{Methods summary}

For experimental data, we performed the meta-analysis on Standardized Means Difference (SMD; Hedges' g). For correlational data, meta-analyses were done on Pearson correlations after Fisher transformations. All estimates were calculated for each factor with the rma.mv function from the metaphor package ${ }^{35}$ in $\mathrm{R}$, which permits to fit meta-analytic multivariate fixed-and random/mixed-effects models with or without factors via linear (mixed-effects) models. Since there were sometimes several measures of HNC and factors in one individual paper and/or one study, we included random effects including lab (multiple data from the same individual paper) and study (multiple data points from the same subjects) to take non-independence of data into account. Since heterogeneity of the true effects within a study (within a lab) could be (or not be) identical, we added a higher-level random effect at the estimate level so that random effects $=\sim 1 / \mathrm{lab} /$ study/estimate.id. Then, we transformed the estimates (SMD and Fisher's Z) into R estimates.

Correlational studies include 9 factors that we organized into 4 broader categories: (1) nature conservation: pro-environmental values (personal interest in and appreciation of nature) and proenvironmental behaviours (self-reported and observed pro-environmental behaviours as well as proenvironmental intentions); (2) human welfare: psychological health, social well-being, physical health and humanistic values (value of human beings including human freedom and progress); (3) lifestyle characteristics: physical contact with real nature (e.g., time spent in nature), environmental education (scientific or naturalist knowledge) and mindfulness practices, and (4) non-environmental values: "materialistic" values (values focusing on wealth, possessions, image, and status including consumerism) and conservatism (political values emphasizing traditional social institutions). 


\section{References}

1. Shukla, P.R, Skea, J., Calvo Buendia, E., Masson-Delmotte, V., Pörtner, H.- O., Roberts, D. C., Zhai, P., Slade, R., Connors, S., van Diemen, R., Ferrat, M., Haughey, E., Luz, S., Neogi, S., Pathak, M., Petzold, J., Portugal Pereira, J., Vyas, J., Huntley, E. P., Kissick, K., Belkacemi, M., Malley, J. IPCC, 2019: Climate Change and Land: an IPCC special report on climate change, desertification, land degradation, sustainable land management, food security, and greenhouse gas fluxes in terrestrial ecosystems. (in press).

2. Watson, R. \& AH Zakri et al. Living beyond our means: Natural assets and human well-being: Statement from the board. Millenium Ecosystem Assessment. (2005).

3. Brondizio, E. S., Settele, J., Díaz, S. \& Ngo, H. T. Global assessment report on biodiversity and ecosystem services of the Intergovernmental Science-Policy Platform on Biodiversity and Ecosystem Services. IPBES Secr. Bonn (2019).

4. CBD (Convention on Biological Diversity). Strategic Plan for Biodiversity 2011-2020, Including Aichi Biodiversity Targets. Convention on Biological Diversity https://www.cbd.int/sp/ (2011).

5. Buchanan, G. M., Butchart, S. H. M., Chandler, G. \& Gregory, R. D. Assessment of national-level progress towards elements of the Aichi Biodiversity Targets. Ecol. Indic. 116, 106497 (2020).

6. Schultz, P. W. The structure of environmental concern: Concern for self, other people, and the biosphere. J. Environ. Psychol. 21, 327-339 (2001).

7. Leopold, A. A Sand County almanac, and sketches here and there. (Oxford University Press, USA, 1989).

8. R.K. Pachauri and L.A. Meyer (eds.). IPCC, 2014: Climate Change 2014: Synthesis Report. Contribution of Working Groups I, II and III to the Fifth Assessment Report of the Intergovernmental Panel on Climate Change. IPCC, Geneva, Switzerland, 151 pp. (2014).

9. Díaz, S. et al. Pervasive human-driven decline of life on Earth points to the need for transformative change. Science 366, (2019).

10. Adenle, A. A. Failure to achieve 2010 biodiversity's target in developing countries: How can conservation help? Biodivers. Conserv. 21, 2435-2442 (2012).

11. Rolston, H. Dominion. (Colorado State University. Libraries, 2013).

12. Collins, B. C. \& Press, B. J. U. Genesis 1: 26-28: Significance and Challenges. (2018).

13. Descartes, R. Discours de la méthode. 46.

14. Kant, I. Idea for a universal history with a cosmopolitan purpose. Cosmop. Read. 17-26 (1784).

15. https://.

16. Miller, J. R. Biodiversity conservation and the extinction of experience. Trends Ecol. Evol. 20, 430434 (2005).

17. Whitburn, J., Linklater, W. \& Abrahamse, W. Meta-analysis of human connection to nature and proenvironmental behavior. Conserv. Biol. 34, 180-193 (2020). 
18. Mackay, C. M. L. \& Schmitt, M. T. Do people who feel connected to nature do more to protect it? A meta-analysis. J. Environ. Psychol. 65, 101323 (2019).

19. Pritchard, A., Richardson, M., Sheffield, D. \& McEwan, K. The Relationship Between Nature Connectedness and Eudaimonic Well-Being: A Meta-analysis. J. Happiness Stud. 21, 1145-1167 (2020).

20. Capaldi, C. A., Dopko, R. L. \& Zelenski, J. M. The relationship between nature connectedness and happiness: a meta-analysis. Front. Psychol. 5, (2014).

21. Rabinowitz, P. M., Pappaioanou, M., Bardosh, K. L. \& Conti, L. A planetary vision for one health. BMJ Glob. Health 3, e001137 (2018).

22. Clay, Z. \& de Waal, F. B. Development of socio-emotional competence in bonobos. Proc. Natl. Acad. Sci. 110, 18121-18126 (2013).

23. Plotnik, J. M., De Waal, F. B. \& Reiss, D. Self-recognition in an Asian elephant. Proc. Natl. Acad. Sci. 103, 17053-17057 (2006).

24. Genty, E., Breuer, T., Hobaiter, C. \& Byrne, R. W. Gestural communication of the gorilla (Gorilla gorilla): repertoire, intentionality and possible origins. Anim. Cogn. 12, 527-546 (2009).

25. Mancuso, S. \& Viola, A. Brilliant green: the surprising history and science of plant intelligence. (Island Press, 2015).

26. Yokawa, K., Kagenishi, T. \& Baluška, F. Anesthetics, anesthesia, and plants. Trends Plant Sci. 24, 1214 (2019).

27. Berenguer, J. The effect of empathy in proenvironmental attitudes and behaviors. Environ. Behav. 39, 269-283 (2007).

28. Atran, S. et al. Folkecology, Cultural Epidemiology, and the Spirit of the Commons: A Garden Experiment in the Maya Lowlands, 19912001. Curr. Anthropol. 43, 421-450 (2002).

29. Moore, R. C. \& Cooper Marcus, C. Healthy planet, healthy children: Designing nature into the daily spaces of childhood. Biophilic Des. Theory Sci. Pract. Bringing Build. Life 385, (2008).

30. Hughes, J., Rogerson, M., Barton, J. \& Bragg, R. Age and connection to nature: when is engagement critical? Front. Ecol. Environ. 17, 265-269 (2019).

31. Liefländer, A. K., Fröhlich, G., Bogner, F. X. \& Schultz, P. W. Promoting connectedness with nature through environmental education. Environ. Educ. Res. 19, 370-384 (2013).

32. Rosa, C. D., Profice, C. C. \& Collado, S. Nature Experiences and Adults' Self-Reported Proenvironmental Behaviors: The Role of Connectedness to Nature and Childhood Nature Experiences. Front. Psychol. 9, (2018).

33. Windhorst, E. \& Williams, A. Growing up, naturally: The mental health legacy of early nature affiliation. Ecopsychology 7, 115-125 (2015).

34. Wilks, M., Caviola, L., Kahane, G. \& Bloom, P. Children prioritize humans over animals less than adults do. (2020). 
35. Viechtbauer, W. \& Viechtbauer, M. W. Package 'metafor'. Compr. R Arch. Netw. Package 'metafor' Httpcran R-Proj. Orgwebpackagesmetaformetafor Pdf (2015).

\section{Methods}

\section{Data search and inclusion}

We followed the PRISMA guidelines to conduct the review. We searched previous papers assessing HNC on Web of Science (WoS) and PubMed from 1900 to 2020 with search terms corresponding to the different HNC scales used in previous published reviews, opinions and meta-analyses (see details in Extended data Table 1) and we obtained 2098 citations (see Supplemental information for detailed methods and Extended data Figure 1 for PRISMA flow diagram). Only empirical, quantitative and peerreviewed published papers, which were written in English were made eligible for inclusion. In addition, we focused on explicit psychological HNC, also called "HNC as mind" ${ }^{1}$, which refers to as validated and repeatable psychometrics questionnaires developed by psychologists, economists and social scientists to measure HNC. Specifically, among the hundreds of HNC measures which have been developed, we focused on the five more widely used scale (for a detailed description of HNC scales see SI Appendix 2 and 3): The New environmental paradigm (NEP) ${ }^{2}$, the Inclusion of Nature in the Self (INS) ${ }^{3}$, Environmental identity scale (EID) ${ }^{4}$, the Connectedness to nature scale $(\mathrm{CNS})^{5}$ and the Nature relatedness scale (NR) ${ }^{6}$. As such, papers measuring at least one widely used and repeatable HNC measure (NEP, INS, EID, CNS and NR) and at least one factor (correlational and/or experimental studies) were included. At the end of the screening, 124 publications met the eligibility criteria. For all studies, we extracted the number of participants, region (Africa, Asia, Europe, North America, South America and Oceania), country, gender ratio (more than $60 \%$ female, between 40 and $60 \%$ Female, less than $40 \%$ female) and age group (below 18 years-old, between 18 and 25 years old, between 26 and 40 years old, more than 40 years old). For correlation studies (107 individual papers), we extracted zero-order Pearson correlations between HNCs and factors while means and standard deviations of HNC metrics between each condition (i.e., treatment vs. control or pre vs. post treatment) were extracted for experimental studies (35 individual papers). When correlations, means or SDs were missing from the paper, we emailed the corresponding author. Among 67 emails sent ( 25 for pdf requests and 42 for additional information), we obtained 32 successful responses (18 pdfs and 14 additional data). Overall, we obtained a total of 1080 effects sizes from 198 studies from 124 individual papers done by 69,763 participants to perform the meta-analyses.

\section{Factors' description from experimental studies}

We reported 6 types of experimental programs that we organized into 2 broader categories (contact with real nature vs. other programs). Most of the contact with real nature programs involved experimental papers focusing on the impact of exposure to real nature (e.g., walk in the forest, outdoor activities, walk in an indoor garden) or a combination of exposure to real nature and environmental education (naturalist, scientific and ecological knowledge about the natural world) while few experiments 
combined exposure to real nature with mindfulness practices. Other programs include environmental education (scientific or naturalist knowledge acquired through classical ecological courses in an indoor setting), virtual nature (videos, documentaries of nature) and mindfulness practicing in an indoor setting.

\section{Factors' description from correlational studies}

We reported 9 factors that we organized into 4 broader categories (nature conservation, human welfare, lifestyle characteristics and non-environmental values; see SI Appendix 4 for detail description). Most of correlational studies examined the relationship between HNC and nature conservation including proenvironmental values (environmental and social values) and pro-environmental behaviours (self-reported and observed pro-environmental behaviours) or focused on human welfare including psychological health (mental health, positive personality traits, academic performance), social well-being (happiness, social and psychological well-being), physical health (vitality, mobility) and humanist values (value of human beings including human freedom and progress, individually and collectively which include social values, community values, moral values and humanist values). Reversed effect sizes were used for negative mental health (e.g., depression), negative personality trait (e.g., neuroticism) and negative physical health (e.g., physical stress). Other papers focused on the impact of lifestyle

characteristics either contact with real nature (e.g., time spent in nature), environmental education (scientific or naturalist knowledge) and mindfulness practices. Few studies focused on nonenvironmental values (i.e., materialistic and utilitarian values (values focusing on wealth, possessions, image, and status including consumerism) and conservatism (political values emphasizing tradition social institutions).

\section{Statistical analysis}

All statistical analyses were performed in the R (4.0) environment. For experimental data, we performed the meta-analyses on the Standardized Means Difference (SMD; Hedges' g). For correlational data, the meta-analyses were done on the Pearson correlations after Fisher's Z transformations. All estimates were calculated for each factors with the rma.mv function from the metaphor package ${ }^{7}$ which permits to fit meta-analytic multivariate fixed- and random/mixed-effects models with or without factors via linear (mixed-effects) models. Since we can have several measures of HNC and factors in one individual paper and/or one study, we included random effects including lab (multiple data from the same individual paper) and study (multiple data points from the same subjects) to take nonindependence of data into account. Since heterogeneity of the true effects within a study (within a lab) could be or not be identical, we finally add a higher-level random effect at the estimate level so that random $=\sim 1$ llab/study/estimate.id. Then, we transformed the estimates (SMD and Fisher's Z) into R estimates. For each meta-analysis, we thus report the number of individual papers $n$, the number of relationships $k$, the number of subjects $s$ and the R estimates with their $95 \mathrm{Cl}$. In addition, $Z$ tests which follow a normal distribution under the null hypothesis were performed in the absence of factors in the models while omnibus tests were conducted for models including factors. Furthermore, we tested whether the variability in the observed effect sizes was larger than one would expect based on sampling 
variability without factors in the model using a Cochran's Q-test (heterogeneity of the sample sizes) as well as whether the variability in the observed effect sizes or outcomes that is not accounted for by the factors included in the model was larger than one would expect based on sampling variability when factors are included in the model (QE-test for residual heterogeneity). Tests of individual coefficients in the model and the corresponding confidence intervals are based on a standard normal distribution, while the omnibus test is based on a chi-square distribution. We thus report (1) $Z$ and its corresponding $p$ values for models without factors, (2) QM (and its corresponding $p$ values) for models with factors. We also report (1) Q and its corresponding p values Qp for models without factors and (2) QE and its corresponding $p$ values QEp for models with factors for heterogeneity. We provided additional details in Extended data (i.e., funnel and forest plots in Extended data Fig. 4 and supplemental data Appendix 6).

As age, nationality and gender are often considered as a covariate to control in studies, we did not include them as factors but we performed appropriate statistics to investigate their impact on effect sizes. For experimental studies, we compare the impact of the experimental programs while controlling for type of comparison (before/after treatment in different group of subjects vs. pre-post treatment in same individuals), gender ratio, age group (Children vs. adults), duration of the program and time of testing (short term vs. retention test) as fixed effects and lab and study as random effects with the rma.mv function. For correlational studies, we investigate the impact of the factors (4 broad categories) and Region while controlling for gender ratio and age (short term vs. retention test) as fixed effects and lab, study and estimate ID as random effects with the rma.mv function (see Supplemental data Appendix 4 and 5).

\section{Methods' References}

1. Ives, C. D. et al. Human-nature connection: a multidisciplinary review. Curr. Opin. Environ. Sustain. 26-27, 106-113 (2017).

2. Dunlap, R. E., Liere, K. D. V., Mertig, A. G. \& Jones, R. E. New Trends in Measuring Environmental Attitudes: Measuring Endorsement of the New Ecological Paradigm: A Revised NEP Scale. J. Soc. Issues 56, 425-442 (2000).

3. Wesley Schultz, P. The structure of environmental concern: concern for self, other people, and the biosphere. J. Environ. Psychol. 21, 327-339 (2001).

4. Identity and the natural environment: the psychological significance of nature. (MIT Press, 2003).

5. Mayer, F. S. \& Frantz, C. M. The connectedness to nature scale: A measure of individuals' feeling in community with nature. J. Environ. Psychol. 24, 503-515 (2004).

6. Nisbet, E. K., Zelenski, J. M. \& Murphy, S. A. The Nature Relatedness Scale: Linking Individuals' Connection With Nature to Environmental Concern and Behavior. Environ. Behav. 41, 715-740 (2009). 
7. Viechtbauer, W. \& Viechtbauer, M. W. Package 'metafor'. Compr. R Arch. Netw. Package 'metafor' Httpcran R-Proj. Orgwebpackagesmetaformetafor Pdf (2015).

\section{Declarations}

\section{Acknowledgements}

This work was supported by the TULIP Laboratory of Excellence (ANR-10-LABX-41) within the framework of the BIOSTASES Advanced Grant, funded by the European Research Council under the European Union's Horizon 2020 research and innovation programme (No 666971). Parmesan, Singer and Barragan-Jason were funded by a French Make Our Planet Great Again award (project CCISS, number ANR-17-MPGA0007). We thank Dr Florian Arendt, Dr. Alexia Barrable, Dr. Nicolette L. Cagle, Dr. Susan Clayton, Dr. Paul W. Dierkes, Dr. Matthias Forstmann, Dr. Christian Martin, Dr. Kirsten McEwan, Dr. Marijn Meijers, Dr. Lisa K. Nisbet, Dr. Helen Perkins, Dr. Miles Richardson, Alyssa San Jose Abrams (M.Ed), Dr. Markus Schwab and Dr. Daniela Sellmann-Risse for providing additional information and data from their studies. We also thank researchers from the https://stat.ethz.ch/mailman/listinfo/r-sig-meta-analysis// mailing list for providing feedback on statistical analysis especially on the implementation of random effects in the meta-analysis.

\section{Author contributions}

G.B.J., C.M, and M.L. designed research; G.B.J. performed research, analysed data and did the first draft of the paper; all authors gave feedback on data analysis and interpretation and wrote the final version of the paper.

\section{Competing interest declaration}

We declare no competing interests.

\section{Additional information}

Supplementary Information is available for this paper.

Correspondence and requests for materials (code and data) should be addressed to Gladys.barraganjason@gmail.com

\section{Figures}




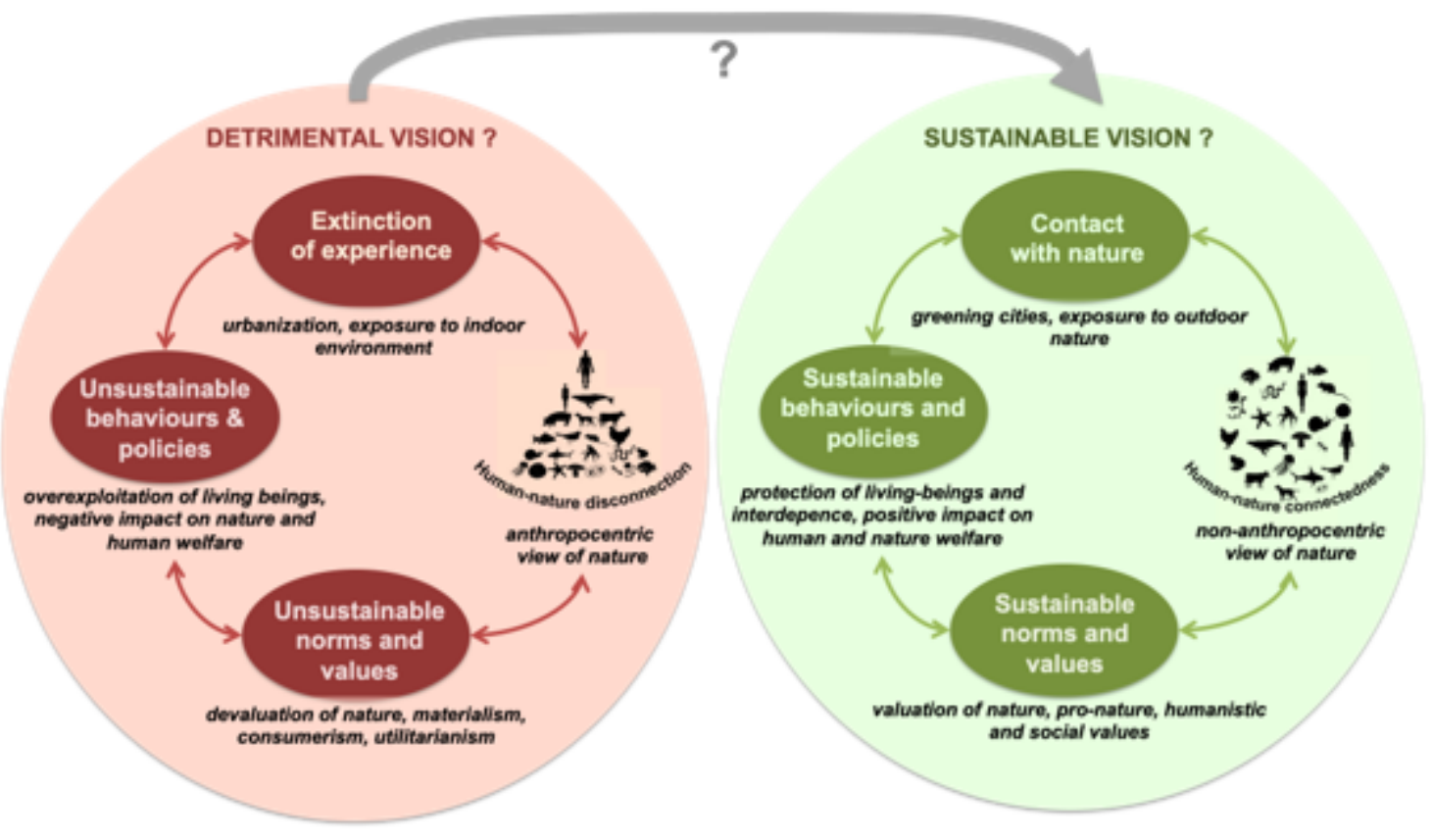

Figure 1

Hypothetical representation of a vicious circle generated by a detrimental worldview (left) and a virtuous circle generated by a sustainable worldview (right). We assess whether and how human-nature connectedness can be used to switch from a detrimental to a sustainable worldview at both individual and societal levels. 


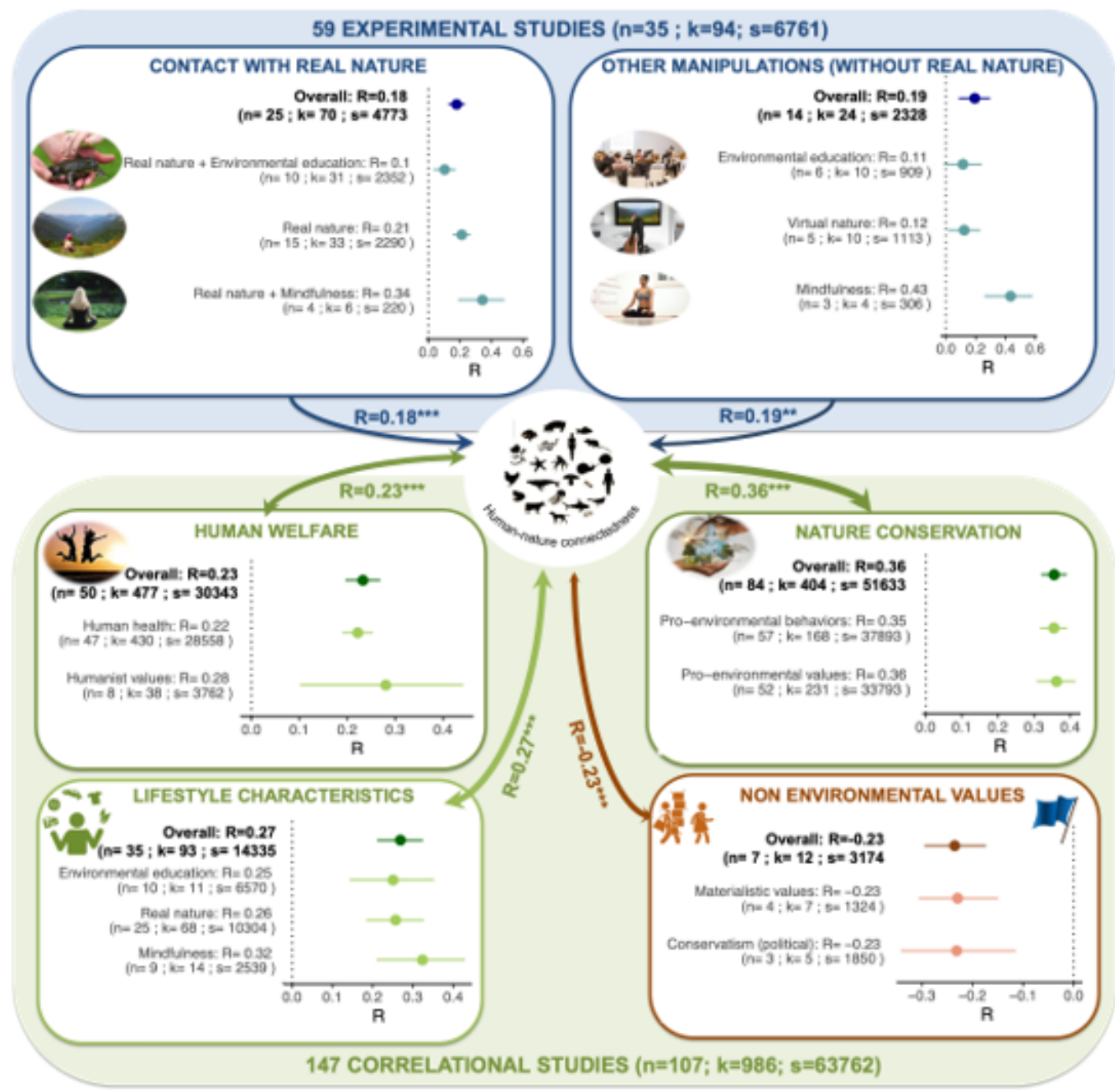

Figure 2

Results from meta-analyses of experimental (blue background and text) and correlational (green and orange backgrounds and text) studies. Each dot represents the average R estimate for each factor obtained after transforming averaged standardized effect sizes (Fisher's Z for correlational data and Hedges' $g$ for experimental data) with $n, k$ and $s$ referring to the number of individual papers, number of effect sizes and number of participants, respectively. Horizontal lines indicate the $95 \%$ confidence intervals for each factor and cross the vertical dot lines when non-significant (i.e., environmental education in experimental studies). Single-headed arrows refer to causal relationships while doubleheaded arrows refer to correlational links. Overall R estimates from each broad category are in bold. Details are provided in Methods summary, Methods, Extended data Tables 2 and 3 and Extended data Fig. 4. Experimental studies show that exposure to nature and mindfulness practices improve HNC. Correlational studies confirmed experimental studies and show that HNC is positively linked to nature conservation and human welfare and negatively linked to non-environmental values. $* *$ : $p<.001 ; * \star \star$ : $\mathrm{p}<.0001$.

\section{Supplementary Files}


This is a list of supplementary files associated with this preprint. Click to download.

- Sl.docx

- Extendeddata.docx 